\title{
Performed Observation Approach Anatomic Site Laterality Code
}

National Cancer Institute

\section{Source}

National Cancer Institute. Performed Observation Approach Anatomic Site Laterality

Code. NCI Thesaurus. Code C95370.

A coded value specifying the laterality of the anatomic location of a performed observation. 